\title{
¿Por qué la simulación en la docencia de las ciencias de salud sigue estando infrautilizada?
}

\author{
Carmen Gomar-Sancho, Jordi Palés-Argullós
}

La simulación es la innovación docente que actualmente despierta más interés, ocupa habitualmente un lugar de honor en los programas de jornadas y actividades de innovación docente, y nos llena la boca y el tiempo de palabras, pero en realidad su uso es extremadamente limitado en la docencia de ciencias de la salud en general, y en medicina y en nuestro país en particular. Se tiene la sensación de que existe un techo de cristal que permite ver posibilidades atractivas y útiles, pero que no alcanzamos.

La simulación se reconoce como una herramienta docente capaz de solventar limitaciones de la metodología docente tradicional y está bien establecida en la formación de profesiones como pilotos, bomberos y trabajadores de centrales nucleares, para formar en seguridad laboral y viaria, en resolución de conflictos, etc. Como cualquier herramienta, puede desarrollarse de muchos modos y utilizarse de forma amplia. Las ciencias de la salud se consideran uno de los campos en los que la simulación tiene más aplicación para la formación del graduado, de especialistas, para la formación continuada y para adquirir las competencias en el trabajo en equipo de distintos profesionales. Entonces, ¿por qué la utilizamos tan poco? Con frecuencia, la queja y el énfasis se ponen en la falta de recursos y de estructura adecuados. A aquellos que aún no han incorporado de forma sencilla, progresiva y natural el uso de la simulación a su tarea docente, los deslumbran los grandes y sofisticados centros de simulación y realidad virtual, dotados de gran estructura, equipamiento y personal especifico muy cualificado, y eso, por supuesto, se ve como inalcanzable o, al menos, alejado de nuestra capacidad individual de utilización. Pero la simulación aplicada a la docencia en ciencias de la salud no debe confundirse con la estructura dedicada a ello; de hecho, los grandes centros de simulación están infrautilizados.

La simulación es una cosa muy sencilla: es la representación artificial de un proceso del mundo real con la suficiente fidelidad para conseguir un objeti- vo específico y que además permite la valoración de la formación o de una determinada acción. En su definición queda claro que debe tener un alto nivel de concreción y circunscribirse a lo que se pretende. No sustituye a la realidad, siempre será limitada en su capacidad de aproximarse a ella, pero nos acerca más que otro tipo de enseñanza. Depende de cuatro elementos: el maniquí o simulador que se utilice, el diseño de la enseñanza, el objetivo del aprendizaje y la capacitación del instructor; es decir, tres de cuatro elementos dependen del profesor. Consideramos muy importante tener esto en cuenta porque creemos que las causas de la infrautilización de la simulación están en razones profundas que tienen que ver con nosotros, los profesores actuales.

En nuestra opinión hay tres barreras que limitan el uso de la simulación por parte del profesorado, por otra parte muy experto ya en la docencia de sus materias. En primer lugar, diseñar enseñanza por simulación precisa una inversión de tiempo inicial del profesor que, probablemente, ya hace años que tiene preparada la enseñanza de ese tema con otra metodología y le da pereza cambiar. En segundo lugar, son todavía relativamente pocos los profesores seniors con suficientes capacidades informáticas desarrolladas para poder aprovechar la disponibilidad de material actual. Y por último, pero muy importante, el profesorado actual no fue enseñado con esta metodología y además ya ha enseñado mucho con otras con las que se encuentra muy cómodo. Por tanto, el pilar del cambio está en un profesorado que quiera innovar, que tenga capacidades informáticas y, desde luego, que se sienta acompañado por un ambiente creado por el centro docente que le facilite avanzar en este campo y que deberá detectar coachers iniciales entre su profesorado.

Sin embargo, aun superando el esfuerzo de la implantación de la enseñanza por simulación, se produce con frecuencia la frustración de algunas expectativas puestas en ella que pueden enfriar el entusiasmo inicial. La simulación no disminuye el tra-
Catedrática de Anestesiología y Reanimación (C. Gomar-Sancho). Catedrático de Fisiología Humana (J. Palés-Argullós). Directores del Laboratorio de Habilidades Clínicas. Facultad de Medicina. Universitat de Barcelona. Barcelona, España.

Correspondencia: Dr. Jordi Palés Argullós. Departamento de Ciencias Fisiológicas I. Facultad de Medicina. Universitat de Barcelona. Casanova, 143. E-08036 Barcelona.

E-mail:

jpales@ub.edu

(c) 2011 Educación Médica 
Tabla. Información necesaria para incorporar la organización de una actividad de enseñanza por simulación a la rutina de un centro.

Asignatura a la que corresponde

Habilidad concreta a desarrollar

Objetivos concretos del taller

Principales errores y complicaciones a evitar

Material necesario, listado con extremo detalle

Necesidad de filmación para el debriefing

Espacio necesario (número de módulos)

Número de grupos al año que realizará el taller

Número de alumnos por grupo

Días de la semana en los que se realizará el taller

Horario

Período

Sistema de evaluación

Valoración de la actitud del estudiante

Valoración global por observación de la realización correcta de la técnica

Valoración paso por paso de la realización de la técnica utilizando listas de comprobación (checklists)

Participación en el debriefing

Profesorado

bajo porque generalmente precisa sesiones ad hoc con presencia del instructor o instructores; no individualiza totalmente la formación, ya que debe hacerse en grupos pequeños y consume tiempo; y se suele realizar una única sesión para cada grupo de alumnos pero no se mantienen los esfuerzos para repetir el entrenamiento o el autoentrenamiento. Es decir, muchas veces el esfuerzo inicial no tiene un resultado a largo plazo y cansa. Además, en este cansancio interviene otro factor, y es que la simulación se considera el mérito de unos pocos y no el deber de todos, y por tanto descansa sólo sobre aquel profesor entusiasta que lo ha puesto en marcha y queda cautivo de su capacidad, no compartida por el resto del profesorado de la materia. Creemos que, hoy en día, el conocimiento de la simulación debería formar parte de las capacidades estándares de un profesor al igual que otras, como por ejemplo la elaboración de presentaciones en Power Point.

Hay que cambiar las actitudes del profesorado y de la organización del centro, y desmitificar la simulación, que debe contemplarse como algo sencillo, cotidiano, integrada como una herramienta más de la metodología docente, fácil de utilizar y, por tanto, organizada de forma rutinaria en nuestro día a día. No tiene que aplicarse a una parte importante del programa, puede hacerse a aspectos muy concretos de éste, en sesiones cortas, de una hora, pero bien estructuradas y con los medios apropiados. Analizando las materias de las ciencias de salud no detectamos ninguna en la que la simulación no tenga un papel docente insustituible con otra metodología. El profesorado de cada asignatura debería analizar su contenido para detectar aquellos campos en los que la simulación tendría beneficios para la enseñanza.

La selección de los medios y el diseño de la sesión hay que hacerlos al inicio, como si se confeccionara un guión de una obra de teatro, consensuado con varios profesores que intervengan en su diseño, que la puedan llevar a cabo indistintamente y que tengan claros los diferentes puntos que se muestran en la tabla. Debe quedar todo por escrito, como una receta para poder ser reproducida por otro y quizá aprovechada en parte para otra materia, en cursos ulteriores, para repetirlo el propio alumno en solitario y para que permita a un personal auxiliar montar el taller sin que al profesor le cueste un esfuerzo adicional hacerlo. Y, quizá la parte más novedosa, debe existir un análisis y discusión final del rendimiento y errores de los alumnos, el debriefing, una de las partes más útiles de la simulación. La simulación tiene un efecto multiplicador de lo que se enseña porque los errores cometidos por los compañeros y el obligado debriefing de cada sesión remarcan la enseñanza.

El profesor puede contar con maniquíes muy sencillos o muy complejos. Hay en el mercado una amplísima oferta, desde los fantomas más simples hasta maniquíes con respuesta fisiológicas. Los propios alumnos pueden explorarse entre sí en muchas de las prácticas, o utilizar técnicas de rol playing en las que los alumnos desempeñen el papel de pacientes, familiares o colegas, y pueden aplicar fantomas a pacientes simulados para compaginar habilidad técnica con otro tipo de capacidades. En fin, las posibilidades sólo están limitadas por la capacidad del profesor de acercarse a la realidad. Pero también hay ya numerosos programas de simulación disponibles en el mercado a precio asequible o gratuito en Internet. Muchas sociedades científicas ofrecen material de gran calidad para el e-learning, de donde el pro- 
fesor de grado puede obtener parte del material necesario. En este momento se dispone de gran cantidad de material en Internet para ser utilizado por el profesor intercalándolo en su material docente, desde pequeñas secuencias de vídeo en sus clases hasta programas de autoenseñanza, de autoevaluación y de evaluación del profesorado. De éstos, los hay de auscultación cardiaca, de reanimación cardiopulmonar, de toma de decisiones en urgencias, de exploraciones endoscópicas, etc., tantos que son innumerables. Con todo este material, el profesor puede incorporar la simulación de una forma integrada en su metodología docente, con poco esfuerzo, pero eso sí, estando informado de su existencia.

La simulación ofrece ventajas para el autoaprendizaje exigido en los nuevos planes de estudio si, una vez diseñada la enseñanza por simulación, se permite al alumno su uso individualizado y libre y la autoevaluación. Para ello, los centros deben trabajar en una organización -sencilla y fácil de controlar- en la que los alumnos acudan a los laboratorios de habilidades y simulación de forma libre, disponiendo de instrucciones para el uso del material y aplicación de la sesión, y actualmente ya con algunos programas de autoevaluación, que además permiten al profesor seguir al alumno vía telemática. Podríamos hablar de una 'simuloteca' en semejanza a una biblioteca.

Los centros docentes, en nuestro caso las facultades, deben actuar como facilitadores e, incluso, como 'desmitificadores' de la utilización de la simulación. Debemos hacer un esfuerzo por no dejar solos a los profesores frente a la aplicación de la simulación. Desde luego, son necesarios unos medios que van desde un emplazamiento físico y un personal técnico mínimo que maneje los equipos y sepa montar cada una de los talleres de simulación programados, hasta un sistema de acceso en todas las aulas a programas de simulación informáticos bien identificados por los profesores de las asignaturas que los utilizan, pero sobre todo hay que crear una plataforma de conocimiento de todo lo que se utiliza por distintos profesores y materias para enseñar por simulación. Se da con frecuencia que cada profesor conoce algunos simuladores o programas de simulación que él ha descubierto y usa, pero ese conocimiento no lo comparte con otros y, por tanto, no se pueden aprovechar los esfuerzos en un camino que nos está costando. Si el centro docente dispone de una plataforma donde cada asignatura, materia o profesor exponga los sistemas de simulación que utiliza, la forma de localizarlos o la descripción de los talleres de simulación que tiene programados, los beneficios para todos serán exponenciales.

Si estas plataformas de cada centro fueran también conocidas y compartidas entre centros, estamos seguros de que el crecimiento exponencial rompería por fin el techo de cristal que creemos que nos impide el avance. 